\title{
Physicists haunted by hints of Higgs boson
}

\section{Alison Abbott}

Weeks from being consigned to history, the Large Electron-Positron collider (LEP) in Geneva has put physicists in a fluster. Suggestions that the LEP has detected the Higgs boson may this week win the collider a onemonth reprieve.

On 14 September, Luciano Maiani, director-general of CERN, the European Laboratory for Particle Physics near Geneva, must decide whether to switch off the LEP at the end of this month as planned. Pulling the plug would deprive CERN of its chance to follow up the intriguing hints that the Higgs particle has indeed been sighted (see Nature 407, 8; 2000).

According to theory, the Higgs boson interacts with subatomic particles to give them mass: the stronger a particle's interaction with the Higgs, the greater its mass. Theorists have predicted that the Higgs itself has a mass of between 50 and 150 giga-electron volts $\left(1 \mathrm{GeV}=10^{9} \mathrm{eV}\right)$, with the greatest probability that the true figure lies somewhere in the middle of this range. By tuning up the LEP's operating energy, CERN scientists earlier this year narrowed the search considerably by showing that the Higgs definitely does not have a mass below $106 \mathrm{GeV}$.

The LEP's successor, the Large Hadron Collider, will run at such extremely high energy levels - $14 \mathrm{TeV}\left(14 \times 10^{12} \mathrm{eV}\right)$ - that it is certain to detect the Higgs, if it exists, even if the particle's mass is as high as $150 \mathrm{GeV}$. CERN is committed to bringing the LHC online by 2005, and construction is due to begin as soon as LEP is switched off.

But the recent results from two of the LEP's four detectors have now thrown these plans into turmoil. In the past few months, the LEP has been tweaked to run at higher

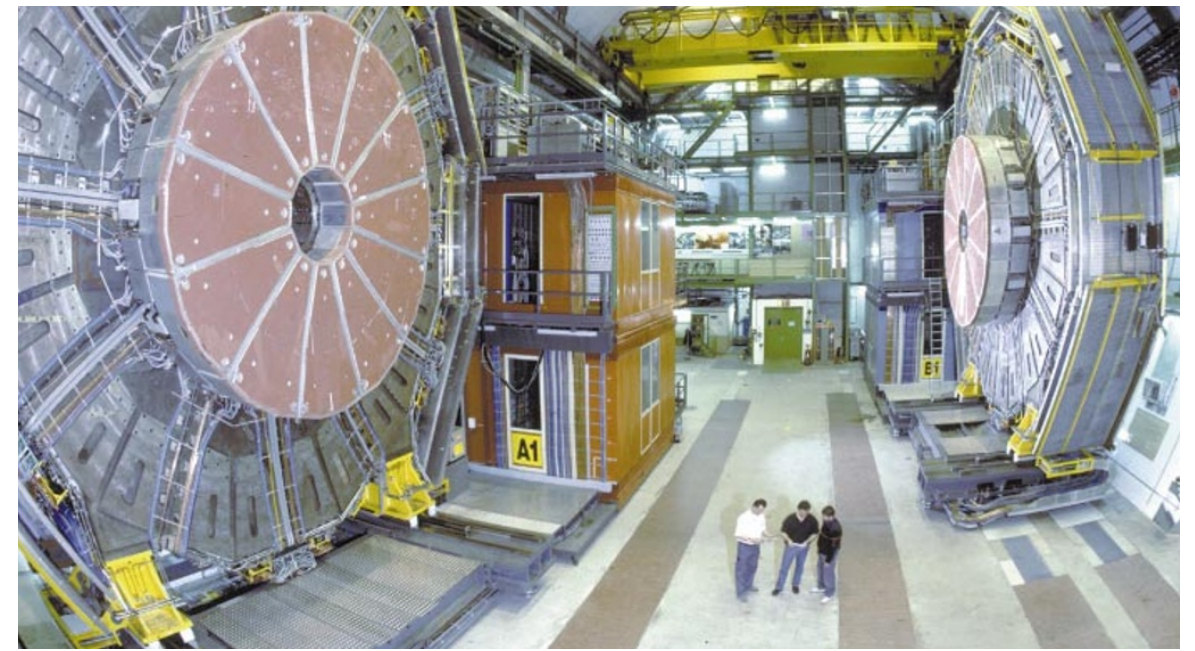

Spotted: the huge detectors of the ALEPH experiment have found possible signs of the Higgs.

energies than ever. "We were not surprised to finally see hints of Higgs at a mass around $115 \mathrm{GeV}$, since this is what theory predicts," says Patrick Janot, LEP physics coordinator. "What surprised everyone was LEP's capability of working at energy levels so much higher than it was originally designed to do: it is a miracle." But for CERN's management, that miracle presents a dilemma, as it is loath to sanction any delays to the LHC.

What the CERN scientists believe they have observed is not the Higgs itself, but its decay products. When the LEP forces an electron and a positron to collide, the resulting energy is converted into matter. Occasionally, this matter will be the Higgs boson, always partnered by the $\mathrm{Z}$ particle. This pair immediately decays to other matter and antimatter particle pairs. According to theory, there is an $85 \%$ chance that the Higgs decays into bottom (b) quarks. But $\mathrm{Z}$ particles are

\section{Gore pledges support for bioscience}

\section{Meredith Wadman, Washington.}

Democratic presidential candidate Al Gore has proposed that, if elected, he will double cancer-research spending over five years and create a new bioengineering institute at the National Institutes of Health (NIH).

An economic plan released on 6 September also calls for an increased emphasis on bioinformatics. It proposes the creation of 20 university-based centres of excellence in biomedical computing.

Gore's plan calls for an $\$ 18$ billion, tenyear increase in spending on "cancer and other medical research" as part of "a larger commitment to all medical research". The National Cancer Institute's current budget is \$3 billion.
The plan does not specify the size of the larger commitment. But Devona Dolliole, deputy national spokeswoman for the Gore campaign, says that Gore plans to double NIH spending on extramural research - an increase of $\$ 83.9$ billion - although she could not say over what time frame.

The 191-page plan says that, along with the cancer money, Gore would provide "similar increases in funding for biomedical research to fight other diseases — from Alzheimer's and Parkinson's to diabetes and HIV/AIDS". His proposal says that a new bioengineering institute at NIH could lead to breakthroughs such as a cure for spinalcord injuries, and production of artificial retinas and kidneys. produced routinely by the LEP and have numerous decay modes. The problem is that $15 \%$ of $Z$ decays also result in b quarks, "thus faking Higgs bosons", says Janot.

High-energy physicists are excited about three b-quark events recorded from the ALEPH experiment and two by the DELPHI experiment, which stand out from the background of Z-particle decays. The experimental teams calculate that there is a $99.5 \%$ chance that the events represent new physics, with observation of the Higgs being the most likely candidate. But for physicists to accept unambiguously that the Higgs has been spotted, the chances of there being another explanation would have to be reduced to one in ten million.

If the LEP were to be run until the end of October, and no more credible events were recorded, then the idea that the Higgs has a mass of $115 \mathrm{GeV}$ would be scotched. If the intriguing events continue to turn up at a similar rate, the chances that there is an explanation other than the Higgs would decrease to a few in 100,000. Janot suggests that there might then be a case for extending the LEP into next year. A one-month extension is quite possible, but sources close to CERN's management dismiss the possibility of any further delays, as that would seriously derail plans for the LHC.

If the LEP did produce a strong suggestion that the Higgs has a mass of around 115 $\mathrm{GeV}$, it would allow physicists working at the Tevatron accelerator at Fermilab, near Chicago, to focus their efforts on the relevant energies. Tevatron is not optimized to searching for the Higgs. "For us, it's more like looking for Higgs in a garbage dump," says Hendrik Weerts of Michigan State University, co-spokesman for the Tevatron's $D_{0}$ experiment. Even so, if the CERN findings are real, he believes the Tevatron could pin down the Higgs within three years - well before the LHC is finished. 